OPEN ACCESS

University of Dundee

\title{
Propagation Elements for the Link Budget of Broadband Satellite Systems in Ka and
} Q/V Band

Ventouras, Spiros; Crawford, Paul S.; Kourogiorgas, Charilaos I.

Published in:

Wireless and Satellite Systems - 9th International Conference, WiSATS 2017, Proceedings

DOI:

10.1007/978-3-319-76571-6_15

Publication date:

2018

Document Version

Peer reviewed version

Link to publication in Discovery Research Portal

Citation for published version (APA):

Ventouras, S., Crawford, P. S., \& Kourogiorgas, C. I. (2018). Propagation Elements for the Link Budget of Broadband Satellite Systems in Ka and Q/V Band. In Wireless and Satellite Systems - 9th International Conference, WiSATS 2017, Proceedings (Vol. 231, pp. 155-162). (Lecture Notes of the Institute for Computer Sciences, Social-Informatics and Telecommunications Engineering, LNICST; Vol. 231). Springer Verlag. https://doi.org/10.1007/978-3-319-76571-6_15

\section{General rights}

Copyright and moral rights for the publications made accessible in Discovery Research Portal are retained by the authors and/or other copyright owners and it is a condition of accessing publications that users recognise and abide by the legal requirements associated with these rights.

- Users may download and print one copy of any publication from Discovery Research Portal for the purpose of private study or research.

- You may not further distribute the material or use it for any profit-making activity or commercial gain.

- You may freely distribute the URL identifying the publication in the public portal.

Take down policy

If you believe that this document breaches copyright please contact us providing details, and we will remove access to the work immediately and investigate your claim. 
This is a post-peer-review, pre-copyedit version of an article published in Wireless and Satellite Systems - 9th International Conference, WiSATS 2017, Proceedings. The final authenticated version is available online at: https://doi.org/10.1007/978-3-319-76571-6_15

\title{
Propagation Elements for the Link Budget of Broadband Satellite Systems in Ka and Q/V Band
}

\author{
Spiros Ventouras ${ }^{1}$, Paul S Crawford ${ }^{2}$, and Charilaos Kourogiorgas ${ }^{1}$ \\ ${ }^{1}$ RAL Space, Harwell, Oxfordshire, UK \\ \{spiros.ventouras, \{ HYPERLINK "mail- \\ to:charilaos.kourogiorgas\%7d@stfc.ac.uk" \} \\ ${ }^{2}$ University of Dundee, Dundee, United Kingdom \\ $\{$ pscesat.dundee.ac.uk\}
}

\begin{abstract}
In this paper we discuss two propagation elements which are crucial in the design - from both economical and technological perspective - of HTS systems utilizing Ka-, Q/V- or even W- frequency bands. These are the statistical combination of rain and cloud attenuation statistics and the interannual variability of the attenuation statistics. Also the recently proposed model for the prediction of annual rain statistics (ITU-R Rec. 837-7) is compared with rain data of 13 consecutive years at Chilbolton, UK.
\end{abstract}

Keywords: HTS systems, total attenuation, Ka band, Q band, inter-annual variability, system risk assessment, Rec 837.

\section{Introduction}

The choice of operating frequency for a satellite radio link is constrained by a number of factors. Some are fundamental physical properties (e.g. bandwidth being limited to a moderate fraction of the chosen centre frequency, tropospheric impairments), while others are constrained by current technology (e.g. cost of ground based hardware, available space-based transmitter power, etc.), and of course the regulatory frameworks in place to manage the use of the radio spectrum. As a general rule, moving to higher frequencies provides greater bandwidth and thus potential data rates but is more demanding in terms of technology (and thus hardware cost), and ultimately more prone to the effects of atmospheric gases, clouds, rain and tropospheric turbulence.

The majority of the atmospheric phenomena exhibits a stochastic behaviour both in time and space and therefore differ from all other deterministic factors (e.g., free space loss) that affect the satellite link under clear sky link conditions. Furthermore, since the power resources are limited and bearing in mind a) the levels of atmospheric attenuation which have to be coped with and $b$ ) the higher performance requirements of these higher frequency systems, more accurate link budget calculations are required. 
This means that the possible fluctuations of the various contributions to the link budget are to be considered to comply with the requirements in all conditions. One cause of these fluctuations is the prediction error of the propagation elements of the link budget (e.g. fade margin at a specific probability level). Therefore this propagation prediction error has to be modelled in order to provide a system risk assessment (i.e. risk of not reaching the availability target) and an indication of the system reliability.

Section 2 of this paper provides the background regarding the concept of the Link Margin, whereas in section 3 the prediction of total attenuation statistics that required for the Link Budget calculations is discussed; a new prediction method is explained. The uncertainty in the system design because of the variability of the annual attenuation statistics is discussed in section 3. Also, in this section, the recently proposed model for the prediction of annual rain statistics (ITU-R Rec. 837-7), required for the prediction of attenuation statistics, is compared with rain data of 13 consecutive years from Chilbolton, UK.

\section{$2 \quad$ Link Budget}

The link performance is evaluated by the parameter Carrier-to-Noise Density ratio $\left(\frac{C}{N_{o}}\right)$ given by the equation [1] :

$$
\left(\frac{C}{N_{o}}\right)=E I R P+\left(\frac{G}{T}\right)-\left(L_{F S}+\sum \text { OtherLoss }\right)+228.6(\mathrm{dBHz} \quad)
$$

Where $C$ is the average RF carrier power, $N_{o}$ the noise power density, EIRP the effective isotropic radiated power $(\mathrm{dBW}),\left(\frac{G}{T}\right)$ the the figure of merit of the receiving terminal $\left(\mathrm{dBK}^{-1}\right), L_{F S}$ the free space loss $(\mathrm{dB}), \sum$ OtherLoss are the additional losses e.g. due to troposphere and 228.6 is the $\mathrm{dB}$ value of the inverse of Boltzmann's constant.

\subsection{Link Margins}

For a link, with $\left\{\right.$ QUOTE $\left.\left(\frac{\mathrm{C}}{\mathrm{N}_{\mathrm{o}}}\right)_{\mathrm{Req}}\right\}$ as the minimum value of the carrier-to-noise density ratio the available margin $\boldsymbol{M}$ is given by the formula:

$$
M=\left(\frac{C}{N_{o}}\right)-\left(\frac{C}{N_{o}}\right)_{\text {Req }}
$$

where $M \geq 0 d B$ for the link to be capable of operation.

Depending on what losses are included in the link budget calculation we have different margins. The simplest case is where we only have the free space loss, leading to the in-vacuum margin:

\{PAGE $\}$ 


$$
M_{V}=\left(\frac{C}{N_{o}}\right)-\left(\frac{C}{N_{o}}\right)_{R e q}=E I R P+\left(\frac{G}{T}\right)-L_{F S}+228.6-\left(\frac{C}{N_{o}}\right)_{R e q}
$$

However, the in-vacuum margin is of little use for a ground station as there is the always present attenuation due to the atmospheric gases, $\mathrm{A}_{\mathrm{G}}$. Measurements have shown [2] than $A_{G}$ experiences a small variability around its mean value and can be consider fixed either equal to its mean value or for a more conservative approach equal to the exceeded level for the annual percentage 99\%. Both these values can be predicted by the ITU-R Rec. 676. So, the simplest useful margin is the "clear sky" margin:

$$
M_{C S}=M_{V}-A_{G}=E I R P+\left(\frac{G}{T}\right)-\left(L_{F S}+A_{G}\right)+228.6-\left(\frac{C}{N_{o}}\right)_{R e q}
$$

Again we require a positive margin for the link to be capable of any operation in the real world. However, we have other sources of attenuation that are variable and models (e.g. ITU-R) the loss for a given link availability $p$ (in \%) based on:

$$
M_{T}=M_{C S}-A_{V T}(100-p)
$$

Where $\mathrm{M}_{\mathrm{T}}$ is the total link margin (taking in to account all losses) and $\{$ QUOTE $\left.A_{R}(100-p)\right\}$ is the excess tropospheric attenuation threshold exceeded for the $(100-p) \%$ of the average year (i.e., the variable part: rain, cloud, scintillation).

The traditional approach for computing a link budget then is to evaluate the engineering and site-related parameters (EIRP, G/T, $\mathrm{L}_{\mathrm{FS}}$ and required $\mathrm{C} / \mathrm{N}_{0}$ ), and then to compute the gaseous attenuation $\mathrm{A}_{\mathrm{G}}$ to yield the clear-sky margin $\mathrm{M}_{\mathrm{CS}}$ from equation (3). Then for a given availability specification $p_{0}$, we can compute the corresponding excess attenuation $A_{v T}$ and from that we have the total margin $\mathrm{M}_{\mathrm{T}}$ for the link using equation (4). If we find that $M_{T} \geq 0 d B$ then we are satisfied that the link will meet or exceed the requirements.

If we find that $M_{T}<0 \mathrm{~dB}$, or $M_{T}$ is significantly above $0 \mathrm{~dB}$, then normally we will change one or more of the engineering parameters to either enable reliable operation (e.g. increasing EIRP if the margin is below $0 \mathrm{~dB}$ ) or to reduce the overall system cost (e.g. reducing EIRP if a substantial positive margin is found).

Therefore, it is clear that the accurate prediction of Total attenuation statistics (i.e., Gaseous and Excess attenuation) is crucial for the optimum design of the satellite system.

\section{Total Attenuation}

The current ITU-R method for the prediction of total attenuation statistics - Rec. P.618-12, Annex 1 section 2.5 - combines the statistics of the individual tropospheric effects on an equiprobable basis. This combination is very convenient from an engineering point of view, however, it is not correct and can lead to the system overdesign $[2,3]$. In particular, it has been proved to be inappropriate for higher frequency or low 
elevation angle systems where the effects from gases, clouds, light rain and scintillation can be significant.

Total attenuation can be considered as the sum of two components: the gaseous attenuation due to oxygen and water vapour, which is always present, and the excess attenuation due to clouds, rain and scintillation. In beacon attenuation measurements it is difficult to distinguish rain attenuation from its associated raining-cloud attenuation. As a consequence rain attenuation prediction models or experimental rain attenuation data include also raining clouds attenuation. Therefore, the combined attenuation due to rain and clouds can be written as the sum of two components: the rain and raining clouds attenuation component, and the non-raining clouds attenuation component.

In contrast to cloud and rain attenuation, gaseous attenuation is always present. Measurements and studies have shown that the spread of gaseous attenuation around its mean value is less than $\sim 1 \mathrm{~dB}$ across Europe for the frequency range of interest [2]. Therefore, this method estimates the total attenuation level exceeded for a given percentage from: the gaseous attenuation $m_{\text {gaseous }}$ (mean or the exceeded level for the annual percentage 99\%), the rain attenuation statistics (which includes the precipitating cloud attenuation), the cloud attenuation statistics (which refers to nonprecipitating clouds) and the scintillation statistics.

Step 1: Rain and cloud attenuation statistics: Obtain the rain and cloud attenuation statistics either from experimental data or prediction procedures and construct the pairs:

$\left\{P_{i}\left(A_{R} \geq a_{i}\right), a_{i}\right\}$ where $P_{i}\left(A_{R} \geq a_{i}\right)$ is the probability the rain attenuation (including the precipitating cloud attenuation) $a_{i}$ is exceeded

$\left\{P_{i}\left(A_{C} \geq a_{i}\right), a_{i}\right\}$ where $P_{i}\left(A_{C} \geq a_{i}\right)$ is the probability the cloud attenuation (only of non-precipitating clouds) $a_{i}$ is exceeded

The values of the attenuation thresholds $a_{i}$ can be determined in steps of $1 \mathrm{~dB}$ (or $0.5 \mathrm{~dB}$ for a better accuracy), $\{0,1,2,3, \ldots$, a_max $\}$. The value a_max is determined by the maximum measurable attenuation in case of experimental data or the attenuation area of interest in case of predictions.

NOTE 1 If the rain or cloud attenuation statistics are given in terms of percentages (i.e., exceedance probability) they can be derived in terms of attenuation thresholds by interpolation (ITU-R Rec. P. 1144).

NOTE 2 The rain statistics can be obtained from the ITU-R Rec. 618.

NOTE 3 Due to the lack of a model for the prediction of non-precipitating cloud attenuation statistics (or more accurately due to the lack of predictions of total columnar content of reduced cloud liquid water only for non-precipitating clouds) the required non-precipitating cloud statistics can be approximated by the ITU-R Rec. 840). This might be established after the validation of the method with experimental data.

Step 2: Gaseous attenuation: Obtain the mean gaseous attenuation $m_{\text {gaseous }}$ from experimental data or prediction procedures (e.g, ITU-R P. Rec. 676)

Step 3: Contribution from rain and cloud attenuation: The probability, $P_{i}\left(A_{R+C} \geq\right.$ $a_{i}$ ), that the attenuation due to rain and clouds, $A_{R+C}$, exceeds the attenuation threshold of $a_{i} \mathrm{~dB}$, is given by the sum of the exceedance probabilities of rain and cloud attenuation for the attenuation threshold of $a_{i} \mathrm{~dB}$.

$$
P_{i}\left(A_{R+C} \geq a_{i}\right)=P_{i}\left(A_{R} \geq a_{i}\right)+P_{i}\left(A_{C} \geq a_{i}\right)
$$


Step3 provides the pairs $\left\{P_{i}\left(A_{R+C} \geq a_{i}\right), a_{i}\right\}$ where $a_{i}$ as defined in step1 (e.g., \{ $0,1,2,3, \ldots$, a_max $\}$ ).

Step 4: The required attenuation exceeded level $a_{R+C}$ due to cloud and rain for a specific percentage of interest $\mathrm{p}$ of an average year can be obtained as in step1 by performing interpolation on the estimated values of probability $P_{i}\left(A_{R+C} \geq a_{i}\right)$ versus $a_{i}$ (p the abscissa value for $a_{i}$ ).

Step 5: Total attenuation $\left(A_{T}(p)\right)$ exceeded for the percentage of interest $p$ :

$$
A_{T}(p)=m_{\text {gaseous }} \sqrt{a_{R+C}(p)^{2}+A_{S}(p)^{2}}
$$

where $m_{\text {gaseous }}$ is the gaseous attenuation, mean value or the exceeded level for the annual percentage $99 \%$ as estimated in step $2, a_{R+C}(p)$ the attenuation level exceeded for the percentage $\mathrm{p}$ due to rain and cloud as estimated in step 4 and $A_{S}(p)$ the attenuation level exceeded for the percentage $p$ due to scintillation either from experimental data or prediction procedures (e.g. ITU-R Rec.618).

The method has been validated against data collected in the Southern of England during the ITALSAT propagation campaign [2] (see Figure 1). In addition the method presented in the document CP(15)16 3J/130,3M/227 : Proposed Modification to ITUR Rec. p.618, Propagation data and prediction methods for the design of Earth-Space telecommunication systems, allocated to SWG 3M-2 in the ITU-R meeting in April 2015. The outcome was: 3M/TEMP/85 Working document towards revision of Rec. ITU-R P.618-11 to be attached to the WP 3M CR.

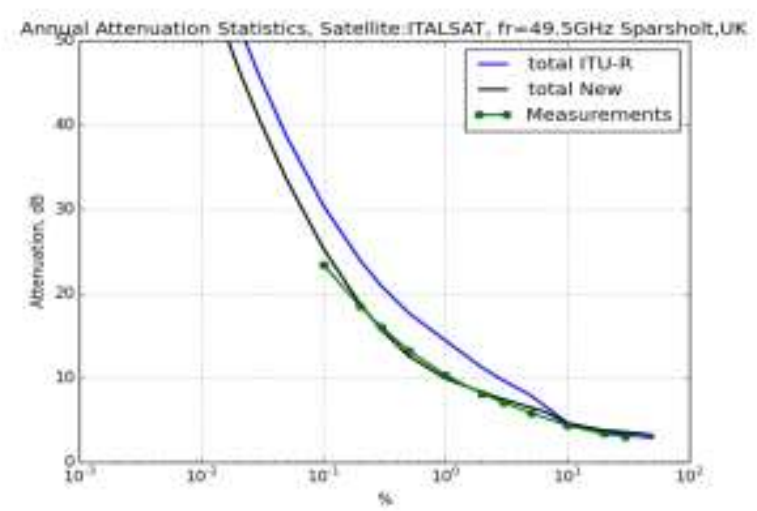

Figure 1. Annual measured and predicted total attenuation statistics at $49.5 \mathrm{GHz}$.

\section{$4 \quad$ Risk Associated with Propagation Margin}

Measurements have shown that a propagation parameter, for example atmospheric attenuation at a given probability level, shows a variability which increases with the decreasing probability. Similar behaviour has been also observed for the rain statistics. As stated in [4] this variability is not to related to climatic variability $[5,6]$ or the 
up-time of an experiment [7]. In fact it can be demonstrated that the statistical estimator used to compute the empirical complementary cumulative distribution function has a significant variance due to the finite duration of the measurements [4].

Figure 2 shows the annual rain rate variability at Chilbolton for consecutive 13 years of measurements in comparison with the ITU-R predictions (Rec. 837), the current (836-6) and the recently proposed modification (837-7).

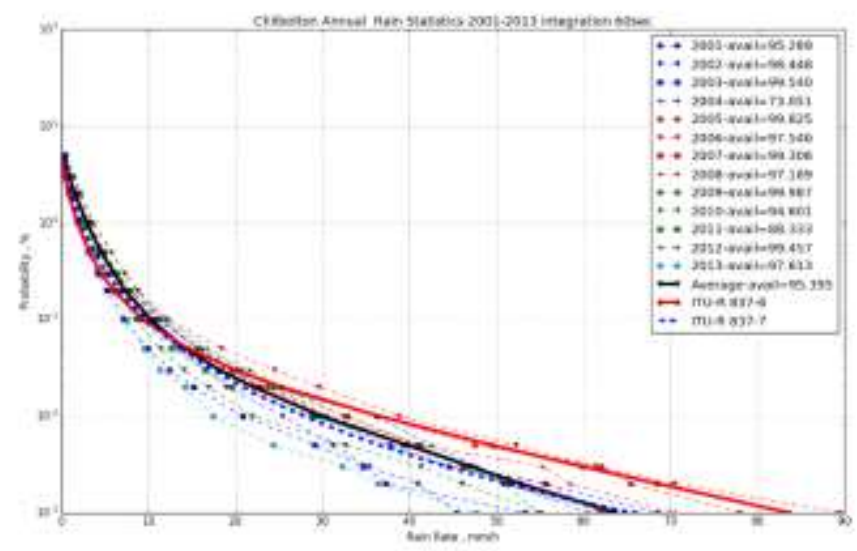

Figure 2. Annual measured and predicted Rain rate statistics at Chilbolton, UK.

The recent ITU-R Rec. P.678-3 (Annex 2) provides a methodology based on study [ 4 ] for estimating the inter-annual variability of rainfall rate and rain attenuation statistics around the long-term Complementary Cumulative Distribution Function (CCDF). In particular, for a given location, the inter-annual fluctuations of rainfall rate and rain attenuation statistics around the long-term Complementary Cumulative Distribution Function (CCDF) $\mathrm{p}$ are normally distributed with mean $p$ and yearly variance $\sigma^{2}$ so that:

$$
\sigma^{2}(p)=\sigma_{C}^{2}(p)+\sigma_{E}^{2}(p)
$$

where $\sigma_{E}^{2}$ is the variance of estimation and $\sigma_{C}^{2}(p)$ is the inter-annual climatic variance.

There is an uncertainty in the system design because of the variability of the annual attenuation statistics. In particular, the total attenuation margin $\mathrm{M}_{\mathrm{T}}$ and consequently the achieved availability Pa depend on the Variable Loss, Avt (see equation 4), which undergoes an annual variability for a given target availability $\mathrm{P}_{\mathrm{T}}$. The system does not meet its specifications when the Variable Loss for a specific year exceeds the estimated value.

From an engineering point of view the following approach is proposed to consider the variability of the annual statistics.

- Estimation of the clear sky Margin $\mathrm{M}_{\mathrm{CS}}$ (equation 4).

\{PAGE \} 
- According to ITU-R Rec. 678 the exceedance probability for the attenuation threshold of $\mathrm{M}_{\mathrm{CS}}$ experiences an inter-annual variability with mean value $p_{c s, m}$ and standard deviation $\sigma_{C S}$ (see equation 8). Estimation of the exceedance probability, $p_{c s, m}$ from the long-term total attenuation statistics (minus the fixed gaseous attenuation ) and the standard deviation $\sigma_{C S}$ (ITU-R Rec. 678).

- For a given target availability $\mathrm{P}_{\mathrm{t}}$ (i.e. exceedance probability $1-\mathrm{P}_{\mathrm{t}}$ ) the system with margin $\mathrm{M}_{\mathrm{CS}}$ meets its specifications if the relevant exceedance probability $p_{c s}$ is less than the target exceedance probability $1-\mathrm{P}_{\mathrm{t}}$. Therefore the probability $\mathrm{P}\left[p_{c s} \leq 1-\mathrm{P}_{\mathrm{t}}\right]$ gives a measure that the system meets its specification whereas the probability $\mathrm{P}\left[p_{c s} \geq 1-\mathrm{P}_{-} \mathrm{t}\right]$ gives a measure of the risk, $\mathrm{R}$, that the system will not meet its specification. Following that the variability of annual attenuation statistics follows the normal distribution ( ITU-R Rec. P.678), the risk $\mathrm{R}$ is estimated from:

$$
R=P\left(p_{C S} \geq 1-P_{t}\right)=\frac{1}{2} \operatorname{erfc}\left(\frac{1-P_{t}-p_{C S . m}}{\sqrt{2} \sigma_{C S}}\right)
$$

Figure 3 illustrates the above procedure.

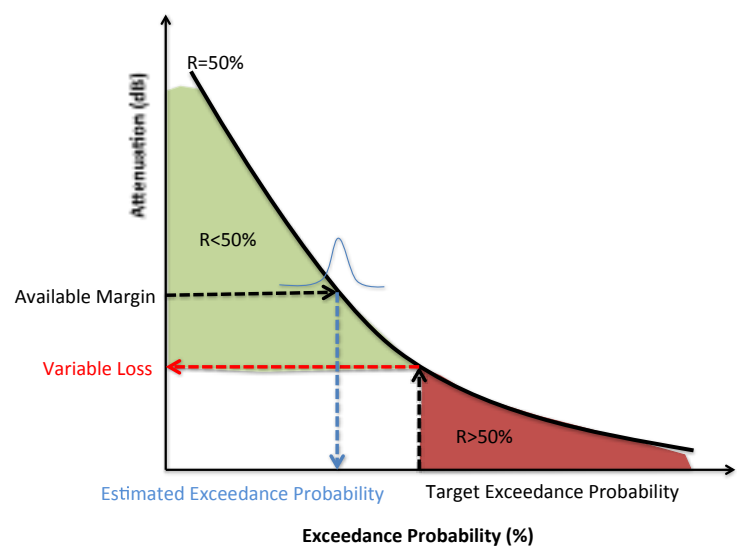

Figure 3. Estimation of the risk associated with propagation margin

\section{Conclusion}

In this paper the prediction of total attenuation statistics that required for the Link Budget calculations was discussed. The current ITU-R prediction it seems that overestimates the total attenuation statistics and can lead to the system overdesign, in particular for $\mathrm{Ka}$ and $\mathrm{Q} / \mathrm{V}$ band systems. A new prediction method based on rigorous mathematical foundations and experimental observations was explained and was in good agreement with the limited, however, experimental data at higher frequencies. 
There is an uncertainty in the system design because of the variability of the annual attenuation statistics. A procedure, based on the ITU-R Rec. 678, was presented in order the variability of the annual statistics to be considered and the risk associated with the system performance to be evaluated.

Also the recently proposed model for the prediction of annual rain statistics (ITU-R Rec. 837-7) was compared with rain data of 13 consecutive years at Chilbolton, UK. The comparison showed a great agreement with the data and a remarkable improvement compared with the Rec. 837-6.

Acknowledgments. This work is funded by the ESA/ESTEC Contract No 4000109354/13/NL/NR "Definition of propagation Elements for System Design of Broadband satellite Systems in Ka and Q/V Band" ( Techical Officer Dr. Antonio Martellucci) and has received funding from the European Union's Horizon 2020 research and innovation programme under the Marie Skłodowska-Curie grant agreement No 665593 awarded to the Science and Technology Facilities Council.

\section{References}

1. Ippolito L. J., "Satellite Communications Systems Engineering: Atmospheric Effects, Satellite Link Design and System Performance", John Wiley, 2008

2. Ventouras S, S.A Callaghan, and C.L.Wrench [2006],"Long-term statistics of tropospheric attenuation from the Ka/U band ITALSAT satellite experiment in the United Kingdom", Radio Science, Vol.41, RS2007

3. Ventouras S and C.L. Wrench, "ITU-R total attenuation predictions in comparison with slant path measurements in southern England"., Electronics Letters , 2002 , DOI:10.1049/el:20020696

4. Jeannin N, Boulanger X, Féral L, Castanet L, Lacoste F. Inter-annual variability, risk and confidence intervals associated with propagation statistics. Part I: theory of estimation, Int. J. Satell. Commun. Network. (2013). DOI: 10.1002/sat.1060

5. J. P. V. Poiares Baptista, Z. W. Zhang and N. J. McEwan, "Stability ofRainRate Cumulative Distributions", Electronic Letters, Vol 22, No. 7,Mar 1986, pp. 350-352

6. Paulson, K. S. (2010), Trends in the incidence of rain rates associated with outages on fixed links operating above $10 \mathrm{GHz}$ in the southern United Kingdom, Radio Sci., 45, RS1011, doi:10.1029/2009RS004193.

7. E. Matricciani, C. Riva, The search for the most reliable long-term rain attenuation cdf of a slant path and the impact on prediction models, IEEE Transactions on Antennas and Propagation, 2005, 53, 3075-3079.

\{PAGE $\}$ 